\title{
Back seeding of picosecond supercontinuum generation in photonic crystal fibres
}

\author{
Moselund, Peter M.; Frosz, Michael Henoch; Thomsen, Carsten; Bang, Ole
}

Published in:

Proceedings of SPIE

Link to article, DOI:

$10.1117 / 12.780965$

Publication date:

2008

Document Version

Publisher's PDF, also known as Version of record

Link back to DTU Orbit

Citation (APA):

Moselund, P. M., Frosz, M. H., Thomsen, C., \& Bang, O. (2008). Back seeding of picosecond supercontinuum generation in photonic crystal fibres. In Proceedings of SPIE: Photonic Crystal Fibers (Vol. 6990, pp. 6990-24). SPIE - International Society for Optical Engineering. https://doi.org/10.1117/12.780965

\section{General rights}

Copyright and moral rights for the publications made accessible in the public portal are retained by the authors and/or other copyright owners and it is a condition of accessing publications that users recognise and abide by the legal requirements associated with these rights.

- Users may download and print one copy of any publication from the public portal for the purpose of private study or research.

- You may not further distribute the material or use it for any profit-making activity or commercial gain

- You may freely distribute the URL identifying the publication in the public portal 


\title{
Back seeding of picosecond supercontinuum generation in photonic crystal fibres
}

\author{
Peter M. Moselund ${ }^{a, b}$, Michael H. Frosz ${ }^{a, b}$, Carsten L. Thomsen ${ }^{b}$, and Ole Bang ${ }^{a}$ \\ ${ }^{a}$ Fibers and Nonlinear Optics Group, DTU Fotonik, Department of Photonics Engineering, \\ Technical University of Denmark, 2800 Kgs. Lyngby, Denmark \\ ${ }^{b}$ Koheras A/S, Blokken 84, DK-3460, Birkerød, Denmark
}

\begin{abstract}
Supercontinuum generation (SCG) has been the subject of intense investigation during the last few years and its main mechanisms are now well understood. Focus has shifted towards tailoring the spectrum of the supercontinuum for specific applications. We experimentally investigate SCG with picosecond pumping in photonic crystal fibers with two closely spaced zero dispersion wavelengths. We couple parts of the output spectrum of the supercontinuum source back to the input in order to produce a gain of over $15 \mathrm{~dB}$ at some wavelengths. We use a variable time delay to optimize the overlap between the pump and the back seeded pulses and investigate how the delay and spectrum of the back seeded pulse affects the resulting supercontinuum spectrum.
\end{abstract}

Keywords: Supercontinuum, continuum, PCF, photonic crystal fiber, parametric gain, soliton, FWM, fourwave-mixing.

\section{INTRODUCTION}

Supercontinuum (SC) generation is a process whereby very intense white light can be generated by high intensity laser light in a nonlinear medium. It has been the subject of intense investigation since the development of photonic crystal fibers (PCFs) a decade ago. With PCFs it became possible to control the guiding properties of fibers with great precision, and therefore broad supercontinua could be produced with much lower peak powers. The technology has now matured to a point where commercial supercontinuum light sources have become available with both ns and ps pumping, such as the SuperK sources from Koheras A/S. Simultaneously the supercontinua have been applied in many fields in biooptics, such as microscopy, ${ }_{1}^{1}$ optical coherence tomography $(\mathrm{OCT}),{ }^{2,3}$ and optical mammography, ${ }^{4}$ and in optical communication for wavelength division multiplexing. ${ }^{5}$ Great progress has also been made in the theoretical study of the complex mechanics of the supercontinuum generation process, and the major effects governing the spectral broadening are now understood. The studies of supercontinuum generation have now moved on to investigating how one can improve the control over the generated spectrum in order to tailor it for specific applications, e.g. strengthening the visible and blue part for fluorescence microscopy or the bands around $800 \mathrm{~nm}$ and $1300 \mathrm{~nm}$ for OCT. ${ }^{2}$ Some of the steps which have been investigated are the use of a two-color pump, ${ }^{6}$ the inclusion gratings in the nonlinear fibre, ${ }^{7,8}$ the use of cascades of different nonlinear fibres, ${ }^{9}$ and variation of the chirp of the pump pulses. ${ }^{10}$

In this work we demonstrate how the spectrum of a supercontinuum source can be modified by feeding part of the output light back into the PCF in which it was generated and time matching it with the pump pulses. We show that in this fashion strong amplification peaks can be created in the supercontinuum.

The concept of utilizing feedback in PCFs has been investigated earlier in several contexts. In the continuouswave $(\mathrm{CW})$ regime, several studies have been made in which an SC was produced by pumping a nonlinear fiber in a ring-cavity setup both with ${ }^{11,12}$ and without ${ }^{12,13}$ a gain medium in the ring. The effect of the feedback loop in these system was a broadening of the spectrum based on a more efficient growth of Raman peaks and a recycling of pump light. However, since all these systems were totally integrated fiber solutions, their maximum possible bandwidth was limited by the bandwidth of the fused fiber components. In a linear cavity scheme, a fiber Bragg

Further author information: (Send correspondence to Peter M. Moselund)

Peter M. Moselund: E-mail: pmm@com.dtu.dk, Telephone: +45 51921492

Photonic Crystal Fibers II, edited by Kyriacos Kalli, Waclaw Urbanczyk,

Proc. of SPIE Vol. 6990, 69900P, (2008)

0277-786X/08/\$18 doi: 10.1117/12.780965

Proc. of SPIE Vol. $699069900 \mathrm{P}-1$ 
grating (FBG) has also been utilized at the input and output of the nonlinear fiber to produce feedback and amplification of the Raman peak. ${ }^{14}$ The result of this approach was that the center of the SC was shifted from the pump wavelength to the Raman gain wavelength. Finally, feedback into an SC has also been used with fs pumping in order to achieve broadband tuneable amplification. ${ }^{15}$ Tuneable local peaks in a broad SC similar to the one reported in this paper have previously been realized by Yeom et al., ${ }^{7}$ who used a long period grating (LPG). However, in that case the generation of the peak was based on the transmission of solitons from a fs pump being modified by the grating, and not on amplification of light reflected back in the fiber, as is the case in the experiment presented in this paper.

One can generally split the applications for SC sources in two main groups. The first group is applications which require a spectrally wide and continuous band of light, such as OCT and frequency combs. The second group is made up of applications where one uses the broad SC together with tuneable filters. Only narrow lines of the spectrum are used but the SC is necessary in order to be able to change the spectral position of the lines. For this second group of applications, tunable peak generation would be preferable to continuum generation, because when a continuum is generated, most of the energy is lost to the wavelengths which are not used. The closest thing to tunable peaks, which have been achieved in PCFs so far, have been peaks based on four-wave mixing (FWM). But in these cases tunability has only been achieved by using a tunable laser as pump ${ }^{15}$ or by changing the fiber. ${ }^{16}$ As supercontinuum sources generate a wide spectrum, it would be much more natural to use the supercontinuum itself as pump. The main reason that this has not been tested before is probably that widely spaced FWM peaks normally only occur in the normal dispersion region, while very broad SC are mainly generated using pumping in the anomalous dispersion. This gives the problem that either one only has a narrow band of wavelengths to feed back or one does not have the widely spaced FWM gain. In either case one cannot use FWM amplification of a seed from the SC itself to produce peaks which can be tuned over a wide range. However, one may be able to solve this problem if one uses a fiber with two closely spaced zero dispersion wavelengths (ZDWs), because it has been shown that while such a fiber can generate a rather wide SC, it also allows phase matching of FWM in a large region. ${ }^{17}$ We will in the following describe how we have produced strong local peaks in a broad supercontinuum generated by feeding back part of the output light from an SC. The SC was generated by pumping with a ps pump in the anomalous dispersion region between two ZDWs.

\section{EXPERIMENTAL SETUP}

\subsection{Measurement setup}

The supercontinuum discussed in this work is generated using a setup which can be seen in figure 1. It is based on a custom built modelocked fiber laser based on Yb-doped polarization maintaining (PM) single mode fiber (SMF) and is passively modelocked using a semiconductor saturable absorber mirror (SESAM). This laser generates a $70.2 \mathrm{MHz}$ pulse-train which is subsequently amplified in two custom built fiber amplifiers based on Yb-doped non-PM SMF. After this initial amplification, the signal is finally amplified using a cladding pumped Yb-doped non-PM double clad fiber amplifier (DCA). The pulse from the oscillator is not transform limited to begin with, and due to self phase modulation (SPM) and chromatic dispersion the pulse has broadened significantly at the output of the DCA. The spectrum of the light from the DCA corresponding to different supercontinuum power levels and the full width half maximum (FWHM) pulse length of the laser can be seen on the right in figure 4 .

The output from the DCA is linearly polarized using a $\lambda / 4$ and a $\lambda / 2$ waveplate before being passed through a free space isolator (OFR IO-5-1064-VHP). The transmission loss of the isolator is $1.5 \mathrm{~dB}$. Beyond the isolator, the polarization with respect to the fiber axis is controlled using another $\lambda / 2$ waveplate. Then the light is coupled into the PCF using an achromatic lens with a coupling efficiency of about $50 \%$. The loss of the PCF is specified by the manufacturer to be $30 \mathrm{~dB} / \mathrm{km}$ at $1000-1100 \mathrm{~nm}$, and most of the pump power remains in this interval, so fiber losses are assumed to be of little importance. Using a $10 \%$ beam splitter, a fraction of the collimated output beam of the PCF is split off, coupled into a fiber, and guided to an ANDO 6315A Optical Spectrum Analyzer (OSA) to be analyzed. The main beam of the output is terminated in a beam dump when single pass measurements are made. For the feedback measurements, the main part of collimated output beam is reflected back into the PCF using the Spectral and delay control (SDC) mirror. The distance between the SDC mirror and the PCF can be varied in order to match the round trip time of the feedback pulse to the period of the pump pulse-train. After the light has been fed back through the PCF, it is collimated by the achromatic lens at the 


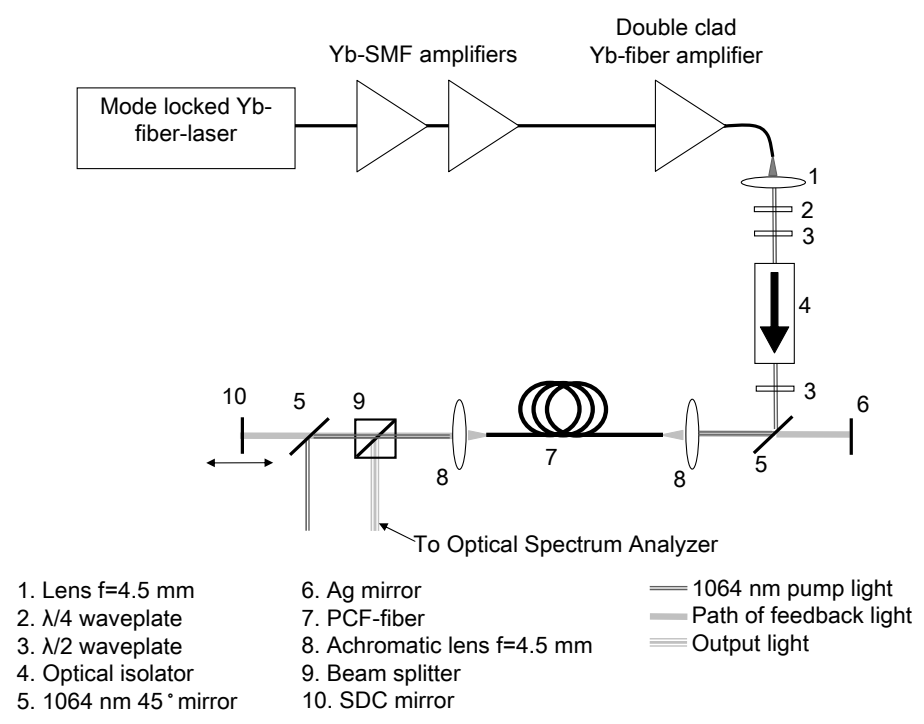

Figure 1. The setup used to produce the feedback and measure the spectrum. The "Spectral and delay control" (SDC) mirror could be altered in order to produce different feedback spectra. The pulse which was fed back was matched in time with pump pulses by tuning the distance between the fiber output and the SDC mirror. Meanwhile the output light was monitored on an Optical Spectrum Analyzer. Mirror 6 was removed and substituted with a fiber going to the OSA or a powermeter head, when the light, which was fed back into the loop, was measured.

fiber input and passed through the $1064 \mathrm{~nm}$ mirror to finally be reflected back by the silver mirror 6 , which thus completes the feedback loop. Note that when the feedback light is being measured in the following, mirror 6 is replaced with a fiber to the OSA. The feedback spectra shown are thus the feedback when it is not interacting with the pump and not the feedback signal present when the entire loop is closed.

\subsection{Fiber}

The fiber used in this work is $4 \mathrm{~m}$ of 1050-Zero-2 fiber commercially available from Crystal Fiber A/S, which is characterized by having two zero dispersion wavelengths (ZDWs) which are close to $1050 \mathrm{~nm}$, separated by a narrow wavelength interval in which the fiber has anomalous dispersion. This can be seen on figure 2 . Since the core diameter of this fiber is only $2.2 \mu \mathrm{m}$, it is very sensitive to variations in the holesize. In order to deduce the dispersion profile of the fiber it was investigated in a scanning electron microscope (SEM) to determine
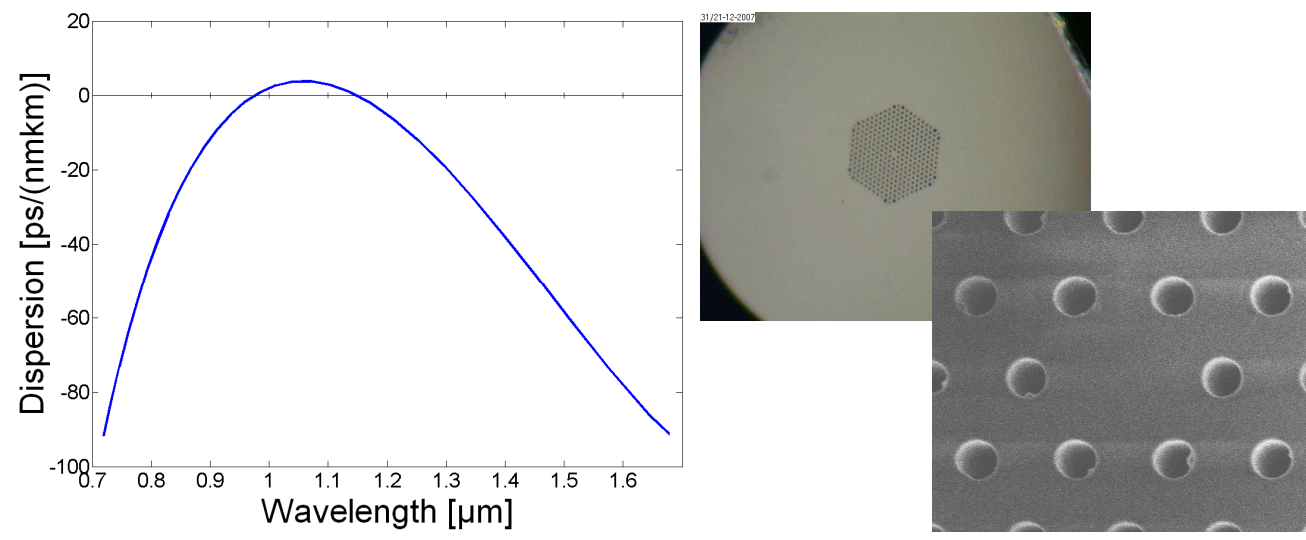

Figure 2. Estimated dispersion profile (left), optical microscope image (upper center) and Scanning Electron Microscope (SEM) image (lower right) of the 1050-zero-2 fiber. The core diameter of the fiber is $2.2 \mu \mathrm{m}$ 
its structural parameters. The dispersion profile was then calculated using the MIT Photonic-Bands (MPB) package. This gave the general shape of the dispersion profile. However, in order to find a more precise value for the dispersion at pump wavelength, the local dispersion was also determined experimentally. This was done using the relationship between the local dispersion and the frequency shift of the MI gain peaks given by Agrawal ${ }^{18}$ to be

$$
\Omega_{\max }= \pm\left(\frac{2 \gamma \mathrm{P}_{0}}{\left|\beta_{2}\right|}\right)^{1 / 2}
$$

where $\Omega_{\max }$ is the angular frequency shift of the maximum gain from the pump peak, $\gamma$ is the nonlinearity parameter of the fiber which is stated by the manufacturer to be $37(\mathrm{Wkm})^{-1}, \mathrm{P}_{0}$ is the peak power, and $\beta_{2}$ is the group velocity dispersion (GVD) parameter with $\mathrm{D}=-\beta_{2} 2 \pi c / \lambda^{2}$.

The the pump wavelength, the spectral position of the modulation instability (MI) peaks, and the movement of the MI peaks as a function of power, all of which can be seen in figure 3, were then entered into equation (1), giving a local dispersion of $4.5 \mathrm{ps} /(\mathrm{nm} \mathrm{km})$. The dispersion curve was then shifted to fit this value. The adjustment of the dispersion curve was made because variations over the fiber length could not be determined from SEM images at a single spot, and the exact holesize was likely to vary. ${ }^{19}$ By calculating the variation in the dispersion profile resulting from small perturbations of the fiber structure, it had been found that small variations in the holesize would mainly alter the position of the dispersion curve, while only modifying the shape slightly. Estimation of the local dispersion from the wavelength and power dependence of the modulation instability (MI) peaks therefore made it possible to correct the calculated dispersion to fit the average dispersion experienced by the light.

It must be mentioned, that although the fiber has not been designed to be birefringent, it was found that the spectrum of the generated supercontinuum was highly dependent on the input orientation of the linearly polarized pump. The spectra which are shown throughout this paper were obtained with a constant pump polarization orientation, which was chosen because it transferred the most energy from the pump line to the SC. The relationship between the input polarization and the axis of the fiber structure has not been investigated.

Similar fibers with two closely lying ZDWs have been investigated previously both numerically and experimentally using fs pulses. ${ }^{2,3,17,20-25}$ However, there is little work on the behavior of PCFs with two ZDWs when longer pulses are used.

\section{EXPERIMENTAL RESULTS}

\subsection{Single pass SC generation}

Initially the spectrum generated in the fiber without feedback was measured as a function of pump power; this can be seen in figures 3 and 4 . The explanation of the evolution of the spectrum is made difficult by the fact that all previous thorough investigations of fibers with closely spaced ZDWs, have been based on fs pulses, where self phase modulation (SPM) has generally been the most important effect. However, as one moves from fs pulses to ps pulses the dynamics generally become much more complicated, because MI induced soliton breakup and soliton collisions make it necessary to consider the contribution and interaction of many solitons. In addition, numerical modeling becomes much more resource intensive as the necessary number of sampling points and fiber-lengths increase substantially. When comparing the measurements to numerical results one also has to consider the uncertainty in determining the actual position of the ZDWs. This is partly because of uncertainty in the measurement and physical parameters used in the numerical fitting, but mainly because the hole-size of the fiber can vary up to $+/-2 \%^{19}$ over the length of the fiber. This means that the ZDWs can vary as much as $+/-50 \mathrm{~nm}$ along the length of the fiber.

The explanation of the supercontinuum generation process in this fiber will be based on previous investigations made of similar fibers in the fs regime, ${ }^{2,17,20-25}$ combined with earlier comparisons between pumping in the fs and ps regime in other fibers. ${ }^{26}$ At low power, only the pump is transmitted with little sign of nonlinear effects, but as the power is increased the pump transmission peak starts to develop shoulders at around $1045 \mathrm{~nm}$ and $1085 \mathrm{~nm}$. As seen in figure 3, these peaks shift to $1035 \mathrm{~nm}$ and $1095 \mathrm{~nm}$ respectively as the power is increased from 46 to $101 \mathrm{~mW}$. The frequency shift of these peaks is approximately proportional to the square root of the pump power. This corresponds to the normal behavior of peaks generated by MI as given by equation 1 . 


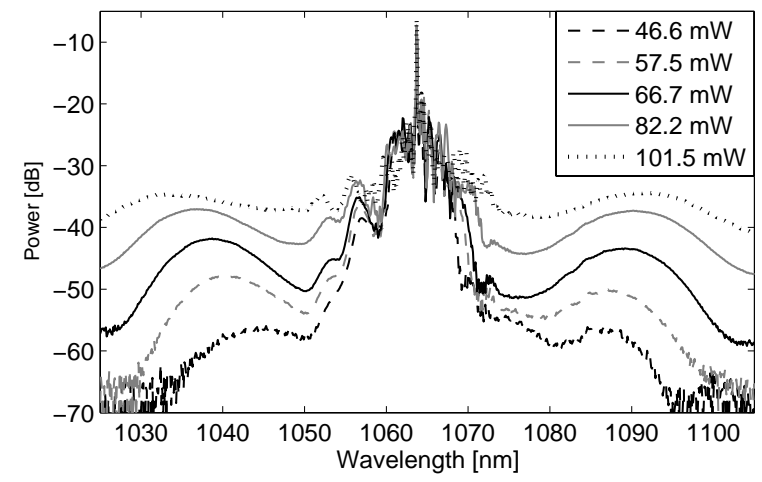

Figure 3. The pump transmission peak at low power levels, where the power dependence of the position of the modulation instability peaks at $1045-1035 \mathrm{~nm}$ and $1085-1095 \mathrm{~nm}$ is evident
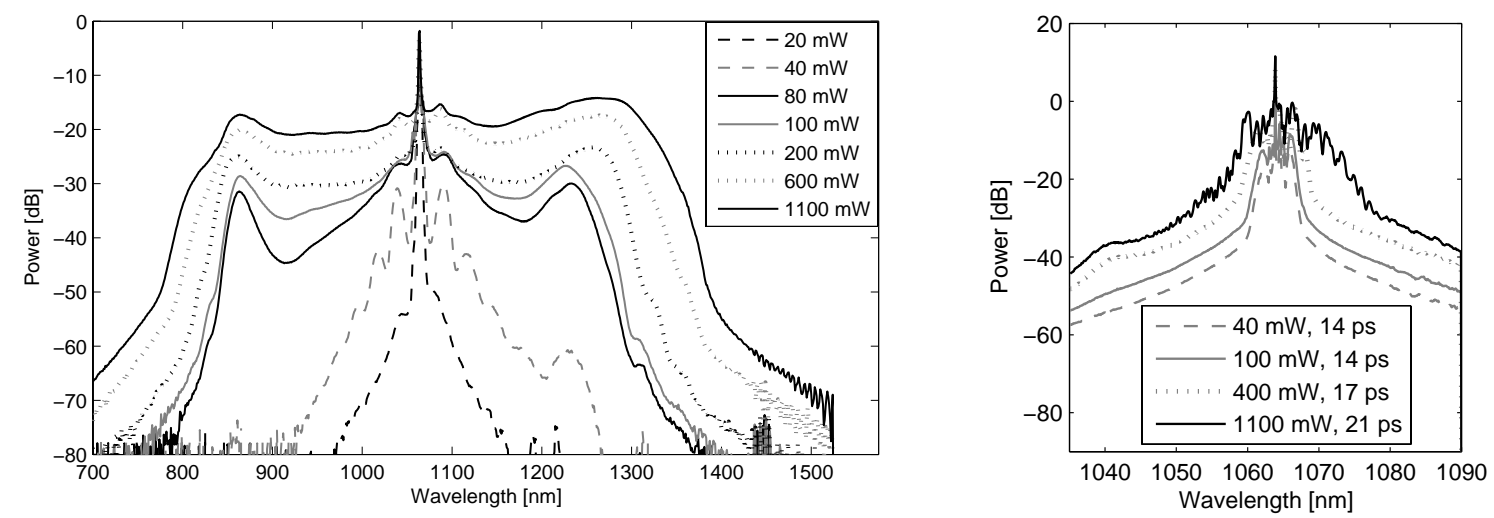

Figure 4. Left: The supercontinuum generated in $4 \mathrm{~m}$ of 1050-zero-2 at different pump average power levels. Right: Spectrum of the pump at various pump powers, measured before the PCF

At higher pump power, a second pair of peaks emerge from the pump around $860 \mathrm{~nm}$ and 1233 , $\mathrm{nm}$ and as the power grows, these peaks broaden and move away from the pump as seen in figure 4. This corresponds to the typical development in an SC pumped by ps pulses in the anomalous dispersion region, where MI causes the pulse to break up temporally and creates a large number of solitons which collide ${ }^{27}$ and create dispersive waves in the normal dispersion region. ${ }^{26}$ We are currently in the process of running numerical simulations using the parameters of the system, and once the results of these are known, a more detailed analysis of the SC generation process will be published elsewhere.

\subsection{Feeding back part of the SC spectrum}

It has been shown in the previous section that the 1050-zero-2 fiber allowed the generation of light in a rather wide spectrum. It is believed that the light is mainly generated by a dispersive wave gain, but it has been shown that fibers similar to this also have FWM phase matching far from the pump. ${ }^{17}$ It was therefore natural to continue the investigation of this fiber by providing a seed for FWM gain by sending back part of the light generated near the FWM phase matching wavelengths and time matching it with the pump pulses. In order for the FWM processes to be efficient this investigation was focussed at an average power of $100 \mathrm{~mW}$, which was one of the highest power levels where the FWM was still clearly an important process in the spectral evolution.

In order to simplify the operation and alignment of the feedback system, a Fabry-Perot cavity design was chosenfor the feedback cavity. The first step was therefore to send part of the output light back through the 


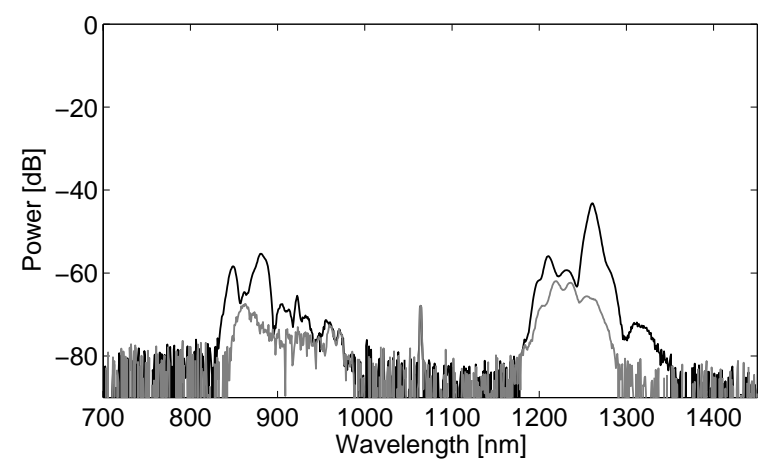

Figure 5. Spectrum of the light traveling backward to mirror 6 with (black) and without (gray) the SDC mirror aligned. The SDC mirror used in this case is a 1200-1700 nm mirror. The multi peaked nature of the spectrum is caused by ripples in the transmission spectrum of the $1064 \mathrm{~nm} 45^{\circ}$ mirrors, which filter out the pump light. The short wavelength peak is caused by unwanted reflection from the 1200-1700 nm mirror because it lacks a broadband anti-reflex coating.

PCF. The spectrum of this feedback light was determined mainly by the reflectance spectrum of the SDC mirror, but also in part by the collimation optics and the transmission of the $1064 \mathrm{~nm} 45^{\circ}$ mirrors which separate the path of the pump from the feedback light. The spectrum which was sent back through the PCF can be seen in figure 5. The gray line shows the light which is reflected back by the fiber facets, even though these have been cleaved at an angle. The black line shows the increased feedback when a mirror is used. When the total output power was $110 \mathrm{~mW}$, the transmission of the $1064 \mathrm{~nm} 45^{\circ}$ mirror which removed the pump at the output to avoid reflecting it back into the pump system was $5.22 \mathrm{~mW}$. Of this, $250 \mu \mathrm{W}$ was coupled back through the fiber to mirror 6 , and it is estimated that $50 \%$ of this was then coupled back into the fiber to be time matched with the pump pulse so that their interaction would alter the output spectrum.

\subsection{Effect of feedback}

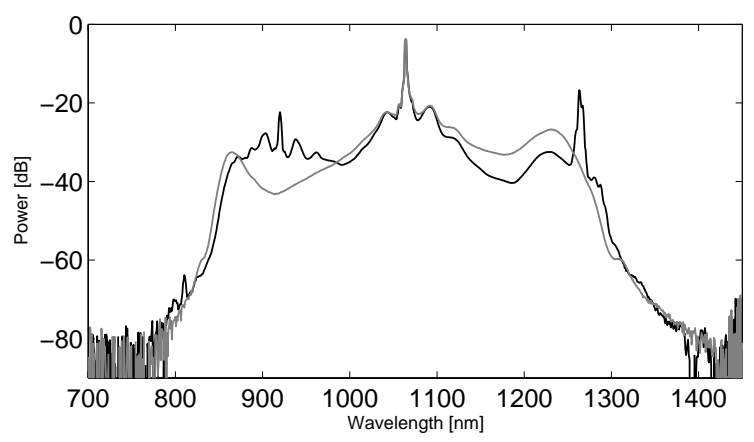

Figure 6. The effect of feedback light in the fiber. Gray: spectrum without feedback. Black: spectrum with feedback

The spectrum of the feedback was first sampled using a fiber at the position of mirror 6 . However, in order to close the feedback loop, mirror 6 was then repositioned and aligned so that the feedback light could be seen to slightly increase the relevant sections of output spectrum with its normal linear contribution. After the spatial alignment was thus completed the temporal matching had to be found by altering the delay distance between the PCF output and the SDC mirror. The delay distance was first roughly tuned using a fast photodiode (Thorlabs D400C) and oscilloscope (Tektronix TDS5052B) in order to match the arrival of the feedback pulse at the output with the arrival of the pump pulse after one round trip. Subsequently, the delay distance was fine-tuned using a micrometer screw to find the strongest amplification of the feedback light. The spectrum generated in the fiber changes significantly when there is time-matched feedback, as can be seen in figure 6 . The most important features are the growth of a $20 \mathrm{~dB}$ peak at the long wavelength end of the spectrum and gains of up to $18 \mathrm{~dB}$ 
in a wide section of the short wavelength side. At the same time, the pump peak and MI shoulders appear to be dampened slightly, while the long wavelength dispersive wave at $1150-1250 \mathrm{~nm}$ is reduced by $5-10 \mathrm{~dB}$. It is important to note that the strongest gain peaks occur at $923 \mathrm{~nm}$ and $1271 \mathrm{~nm}$ and that the sum of the energy of photons corresponding to these two wavelengths is just $0.5 \%$ less than the energy of two photons of the pump wavelength. This is a strong indication that the gain is caused by FWM with the pump. The FWM process removes energy from the pump, which reduces the efficiency of normal SC generation processes, and this may explain the reduction of power at 1150-1250 nm. The FWM, which creates the sharp long wavelength peak, also causes the broad region of peaks in the shorter wavelength end of the spectrum. As can be seen, the short wavelength gain is a broad region centered around a central peak with two side peaks. This closely corresponds with what one would expect if a single strong peak in the long wavelength region had a parametric gain derived from the broad region of the pump and its side peaks.

The fact that such a wide section of wavelengths show a significant amplification at the short wavelength side indicates that the region of phase matching with at least part of the pump and the MI peaks is very wide. It is therefore expected that if one were to feed back a narrow tuneable line of the spectrum instead of the fixed broad reflection used so far, one would be able to generate a peak which was tuneable over a significant interval of wavelengths. This may be an attractive method by which to create a light source which is tunable in a local region, where the general regime of tunability can be chosen by altering the fiber and feedback.

\subsection{Varying the feedback delay}

In order to investigate the length of the temporal overlap between the pump pulse and feedback pulse, which resulted in an amplification, the length of the delay arm was altered slightly while the spectrum was monitored. In this fashion the spectrum as a function of temporal delay between the pump pulse and the feedback pulse could be measured, and this has been plotted in figure 7. The spectrum at a few sample delays can be seen in figure 8.

The most noticeable characteristic of the temporal gain interval is that the strongest effect occurs at 0 ps delay. The 0 ps delay point was set to be the exact delay distance at which the fast diode measurements showed that the feedback pulse and the pump pulse were exactly overlapping at the beam splitter after the PCF. However, one would expect that the greatest effect of the feedback would occur when the feedback pulse was temporally matched with the pump at the input of the PCF. The fact that the greatest effect of feedback coincides with the temporal overlap at the output indicates that the temporal overlap at the output occurs with the same delay as the temporal overlap at the input and thus that the group velocity of the feedback pulse must closely match the velocity of the pump pulse. A close matching of group velocities is a requirement for efficient FWM in a pulsed setup, and the fact that the gain occurs at the point when the pulses overlap at the output is therefore a sign that the gain could be caused by a FWM process.

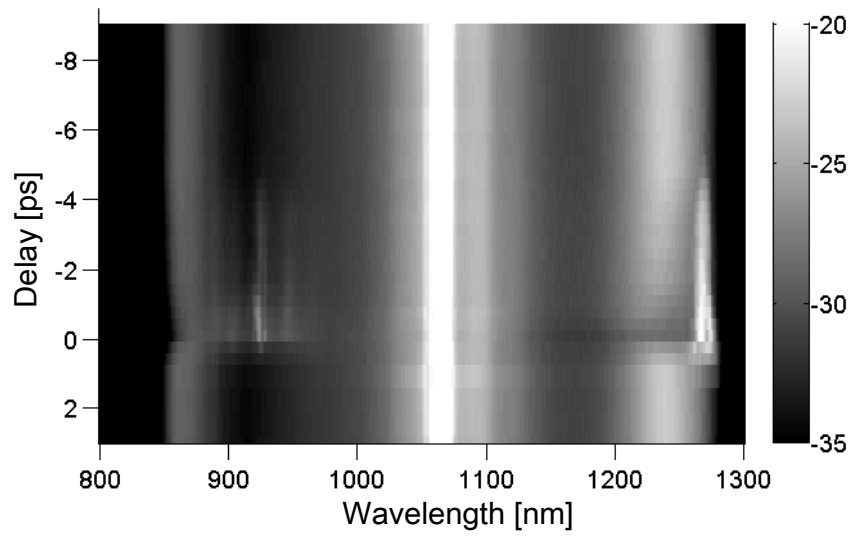

Figure 7. Spectral intensity of the output light with feedback as a function of the delay of the feedback light. The intensity in $\mathrm{dB}$ is indicated by the grayscale on the right. The Y-axis shows the delay, relative to the delay at which the pump pulse was matched with the output pulse at the output beam splitter. 

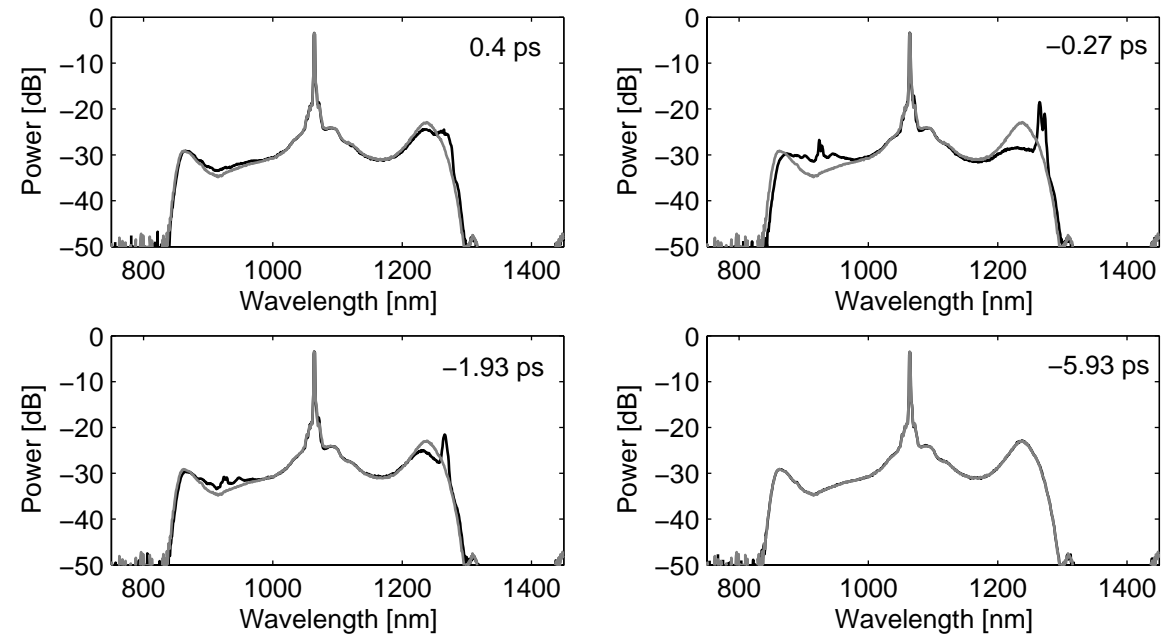

Figure 8. Spectra measured with different feedback delays corresponding to slices in the plot on figure 7 . Black lines show the spectrum with feedback, gray lines without feedback. The lower right plot shows the effect of feedback without time matching.

\subsection{Varying the feedback spectrum}

In order to better understand which wavelengths played a dominant role in the FWM processes, the feedback system was tested using three different mirrors for the feedback, as can be seen in figure 9 . The mirrors were: the 1200-1700 nm mirror, which has been used for all other measurements and which mainly fed back the long wavelength peak of the SC (Figure 9 left), a silver mirror which fed back both sides of the SC (figure 9 center) and finally a broad mirror with reflection centered around $780 \mathrm{~nm}$ which mainly fed back the short wavelength peak
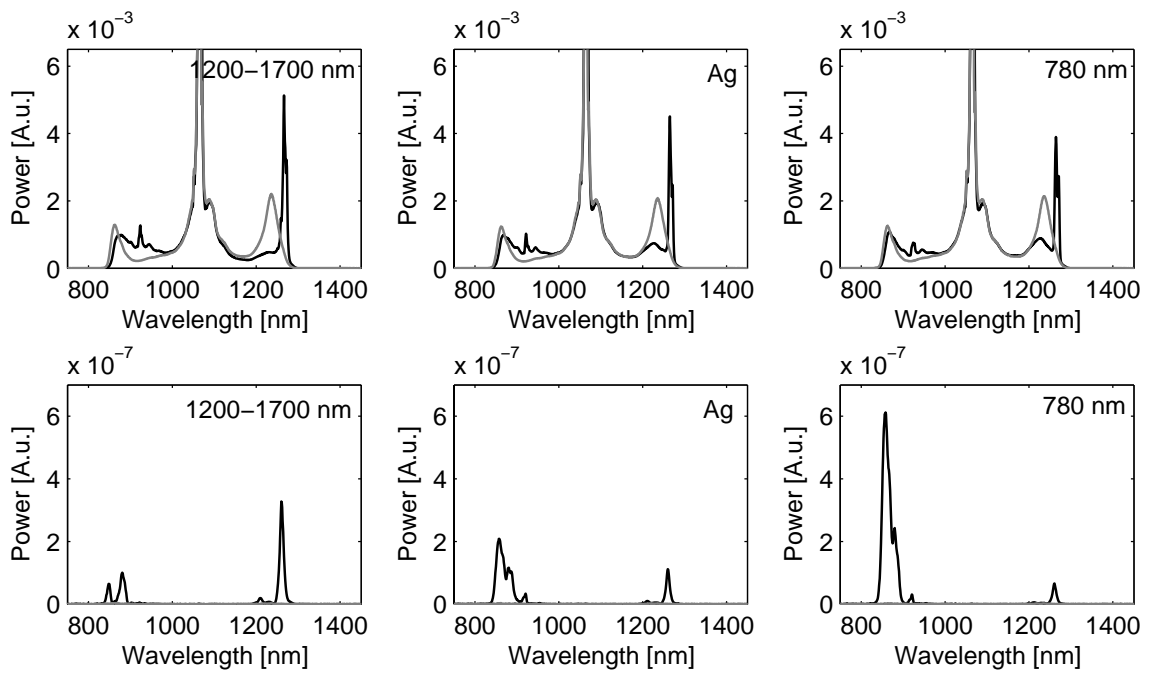

Figure 9. SC spectra generated using various feedback spectra. The three columns correspond to the spectra produced using a 1200-1700 nm mirror (left), an Ag mirror (center), and a broad spectrum mirror centered at $780 \mathrm{~nm}$ (right) as the SDC mirror. The top row shows the output spectrum from the PCF with (black) and without (gray) feedback, while the bottom row shows the spectrum which is fed back through the system, measured at mirror 6 in the setup. Only the light being fed back with the SDC mirror is shown, because the reflection from the fiber facets alone is too small to be seen on a linear scale. Note that all these spectra are plotted on a linear scale. 
of the SC (figure 9 right). The measurements show that as the size of the long wavelength peak is decreased, the amplified long wavelength peak in the output also decreases, although the decrease is not linear with the decrease in the feedback peak. However, the short wavelength peak in the output does not scale with the feedback power at short wavelengths. Instead, the short wavelength increases with the long wavelength amplification peak. This indicates that it is created by the FWM of the long wavelength peak and the pump, instead of being a direct amplification of the feedback at the short wavelengths.

\subsection{Feedback at higher power levels}

All measurements on the effect of feedback in the above have been made with a constant average pump power of about $115 \mathrm{~mW}$, because the greatest relative increase in power at the feedback wavelength occurred at this pump level. In order to get a better understanding of the dynamics and limits of the amplification process, the signal with feedback was measured at a number of pump powers between $20 \mathrm{~mW}$ and $1100 \mathrm{~mW}$ average power, and the resulting spectra can be seen on figure 10 .

At very low power level there is no effect of the feedback, but this is because the pump does not undergo sufficient broadening for there to be any significant light fed back through the fiber. As the power increases above $80 \mathrm{~mW}$, the pump broadens enough to create a significant feedback spectrum, and this is then amplified in the fiber. In the interval between 80 and $200 \mathrm{~mW}$ the increase in power due to feedback is most evident, as the feedback is now sufficiently powerful to be amplified effectively, but the other spectral broadening processes, which are unaffected by feedback, are still weak. After this the long wavelength feedback peak continues to be present and the power of the peak is also rising, but the spectrum around the peak is rising faster than the peak because the normal spectral broadening processes are becoming more and more efficient with increasing pump power. It may also be that the gain peak for FWM gain from the pump is shifting to wavelengths further from the feedback peak, and that could be the reason for the decrease in amplification. The amplification at the short wavelengths only give rise to defined peaks just around $100 \mathrm{~mW}$ pump and thereafter the short wavelength amplification results in an increase in power over the entire region between the pump peak and the short wavelength end of the spectrum. This means that the sharp $1270 \mathrm{~nm}$ peak must be the product of FWM from a very wide region, because otherwise it should be accompanied by a correspondingly narrow low wavelength peak for the sum of the photon energies to add up. Otherwise the $1270 \mathrm{~nm}$ peak must be created by some other process, such as dispersive wave gain, and then be only amplified further by FWM phase match with the pump when the power is around $100 \mathrm{~mW}$.

At all power levels the long wavelength peak arising from the feedback amplification is fixed and corresponds to the wavelength at which there is a peak in the feedback spectrum. If the amplification wavelength had been decided simply by the wavelength at which the pump had the greatest FWM gain, the position of the peak would
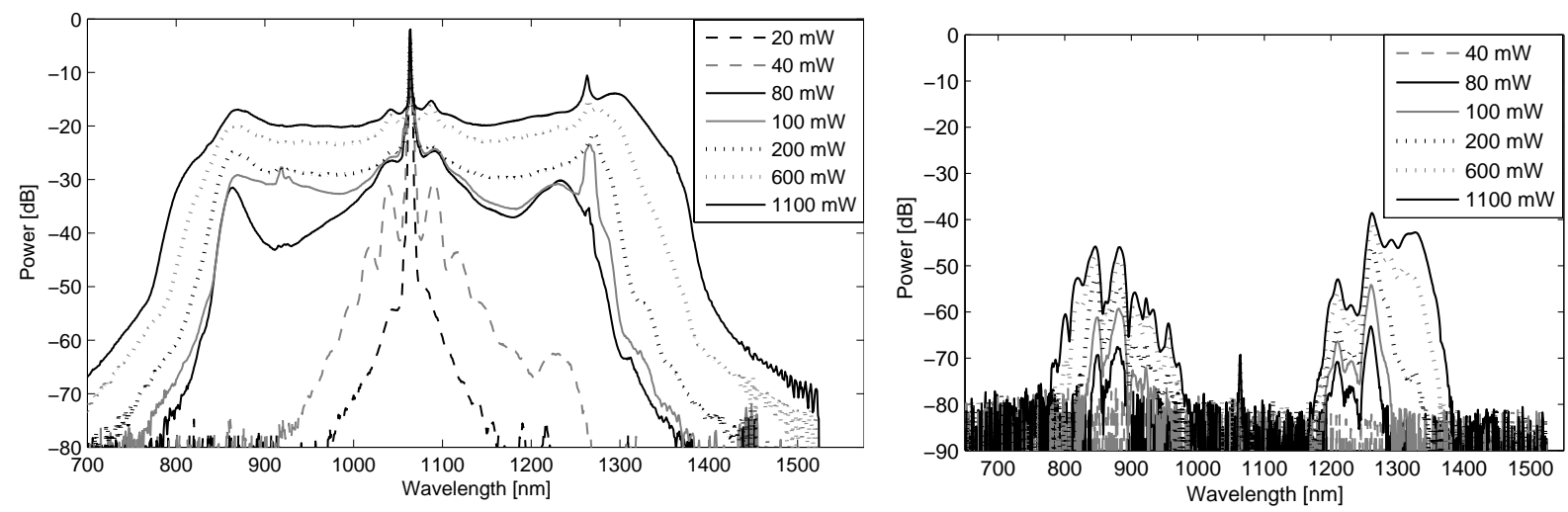

Figure 10. Left: The spectrum of the supercontinuum with feedback at a selection of power levels. Average transmission power is noted in the legend and the spectral power is plotted in dB. Right: The feedback spectra generating the amplification peaks. 
have moved as a function of increasing power, due to the nonlinear part of the phase matching criteria. The fact that the peak is stationary and corresponds to the most powerful wavelength in the feedback shows that the wavelength is controlled by the SDC mirror. This indicates that the position of the peak can be tuned simply by tuning the reflection of the external feedback mirror. Though it is difficult to see on figure 10, the decrease of the $1270 \mathrm{~nm}$ peak in the output at high power levels is actually accompanied by the growth of a wide weak amplification region at longer wavelengths corresponding to longer wavelengths in the feedback spectrum. The fact that gain also occurs at these wavelengths indicate that the tuning region for the peak covers at least several tens of nanometers.

\section{CONCLUSION}

In this work it has been shown that one can significantly modify the spectrum of a supercontinuum source by reflecting part of its output light back into the PCF fiber and time matching it with the pump pulses. It has been shown that strong local amplification peaks of up to $20 \mathrm{~dB}$ can be created, as well as wide areas of over 100 $\mathrm{nm}$ with moderate amplification of a few $\mathrm{dB}$. The presence and position of the amplification can be controlled, at least to some degree, by controlling the spectrum of the light which is sent back into the PCF fiber.

Even if one does not wish to utilize the control over the spectrum that a feedback loop can give, this effect can be important to be aware of because it can appear accidentally if the facets of the PCF are not angle cleaved and its length gives it a round trip time which exactly equals a whole number of pulse periods. The effect will also be a major factor if one uses long ns pulses with short PCFs, in which the pulse is longer than the round-trip time for the light in the fiber, and the pulse thus can overlap temporally with its own reflections.

The most important difference between this work and earlier investigations of parametric gain in PCFs is that earlier works have either used tunable lasers to control the position of the generated peak or simply let the fiber generate the peak where the phase matching and FWM gain spectrum gave the highest gain. In contrast, this method ensures an external control over what light is generated without requiring the inclusion of a complicated tunable laser in the system. The most important conclusion is that previously one has had to choose between pumping in the normal dispersion region in order to get widely spaced parametric gain regions or pumping in the anomalous dispersion region in order to generate a wide supercontinuum. Here we show that it is possible to get both widely spaced FWM peaks and a broad supercontinuum by using mixing between the pump in the anomalous dispersion region and a signal wavelength in the normal dispersion region above the second ZDW. This opens a new area for the shaping of SC spectra in which strong amplification peaks can be created at will inside a wide supercontinuum simply by adding mirrors outside the fiber. However, more research is necessary in order to determine the limits of this technique.

\section{ACKNOWLEDGMENTS}

This work was funded by Photonics Academy Denmark. We thank Crystal Fiber A/S for a fruitful dialog on determining fiber dispersion and for providing the 1050-zero-2 fiber used. Finally the authors would like to thank Lars Hagedorn Frandsen, DTU Fotonik, for providing the scanning electron micrograph image of the fiber.

\section{REFERENCES}

1. J. H. Frank, A. D. Elder, J. Swartling, A. R. Venkitaraman, A. D. Jeyasekharan, and C. F. Kaminski, "A white light confocal microscope for spectrally resolved multidimensional imaging," Journ. of Microscopy 227(3), pp. 203-215, 2007.

2. A. D. Aguirre, N. Nishizawa, J. G. Fujimoto, W. Seitz, M. Lederer, and D. Kopf, "Continuum generation in a novel photonic crystal fiber for ultrahigh resolution optical coherence tomography at $800 \mathrm{~nm}$ and 1300 nm," Opt. Express 14(3), pp. 1245-1160, 2006.

3. P. Falk, M. H. Frosz, O. Bang, L. Thrane, P. E. Andersen, A. O. Bjarklev, K. P. Hansen, and J. Broeng, "Broadband light generation around 1300nm through spectrally recoiled solitons and dispersive waves," Opt. Letters (Early posting), 2008.

4. A. Bassi, L. Spinelli, A. Giusto, J. Swartling, A. Pifferi, A. Torricelli, and R. Cubeddu, "Feasibility of white-light time-resolved optical mammography," Journ. of Biomedical Optics 11(5), p. 54035, 2006. 
5. J. H. Lee, K. Lee, Y.-G. Han, S. B. Lee, and C. H. Kim, "Single, depolarized, CW supercontinuum-based wavelength-division-multiplexed passive optical network architecture with C-band OLT L-band ONU, and U-band monitoring," Journ. of Lightwave Technology 25(10), pp. 2891-2897, 2007.

6. E. Räikkönen, G. Genty, O. Kimmelma, M. Kaivola, K. P. Hansen, and S. C. Buchter, "Supercontinuum generation by nanosecond dual-wavelength pumping in microstructured optical fibers," Opt. Express 14(17), pp. 7914-7923, 2006.

7. D.-I. Yeom, J. A. Bolger, G. D. Marshall, D. R. Austin, B. T. Kuhlmey, M. J. Withford, C. M. de Sterke, and B. J. Eggleton, "Tunable spectral enhancement of fiber supercontinuum," Opt. Letters 32(12), pp. 16441646, 2007.

8. P. S. Westbrook, J. W. Nicholson, and K. S. Feder, "Light generation beyond a continuum edge using a fiber bragg grating," IEEE OFC/NFOEC 2007 Proceedings (OTuJ5).

9. J. C. Travers, S. V. Popov, and J. R. Taylor, "Extended blue supercontinuum generation in cascaded holey fibers," Opt. Letters 30(23), pp. 3132-3134, 2005.

10. C. Cheng, X. Wang, Z. Fang, and B. Shen, "Enhanced dispersive wave generation by using chirped pulses in a microstructured fiber," Opt. Commun. 244, pp. 219-215, 2005.

11. J. H. Lee, K. Katoh, and K. Kikuchi, "Experimental investigation of continuous-wave supercontinuum ring laser composed of clad-pumped Er/Yb codoped fiber and highly-nonlinear optical fiber," Opt. Commun. 266, pp. 281-286, 2006.

12. J. H. Lee and K. Kikuchi, "Experimental performance comparison for various continuous-wave supercontinuum schemes: ring cavity and single pass structures," Opt. Express 13(13), pp. 4848-4853, 2005.

13. W. Zhang, Y. Wang, J. Peng, and X. Liu, "Broadband high power continuous wave fiber Raman source and its applications," Opt. Commun. 231, pp. 371-374, 2004.

14. M. Feng, Y. G. Li, J. Li, J. F. Li, L. Ding, and K. C. Lu, "High-power supercontinuum generation in a nested linear cavity involving a CW Raman fiber laser," IEEE Photonics Technology Letters 17(6), pp. 1172-1174, 2005.

15. Y. Deng, Q. Lin, F. Lu, G. P. Agrawal, and W. H. Knox, "Broadly tunable femtosecond parametric oscillator using a photonic crystal fiber," Opt. Letters 30(10), pp. 1234-1236, 2005.

16. W. J. Wadsworth, N. Joly, J. C. Knight, T. A. Birks, F. Biancalana, and P. S. J. Russell, "Supercontinuum and four-wave mixing with Q-switched pulses in endlessly single-mode photonic crystal fibres," Opt. Express 12(2), pp. 299-309, 2004.

17. K. M. Hilligsøe, T. V. Andersen, H. N. Paulsen, C. K. Nielsen, K. M. R. Kristiansen, K. P. Hansen, and J. J. Larsen, "Supercontinuum generation in a photonic crystal fiber with two zero dispersion wavelengths," Opt. Express 12(6), pp. 1045-1054, 2004.

18. G. P. Agrawal, Nonlinear Fiber Optics, Academic Press, San Diego, California, 3rd ed., 2001.

19. T. T. Alkeskjold, "Personal communication," Crystal Fibre A/S , 2008.

20. M. H. Frosz, P. Falk, and O. Bang, "The role of the second zero-dispersion wavelength in generation of supercontinua and bright-bright soliton-pairs across the zero-dispersion wavelength," Opt. Express 13(16), pp. 6181-6192, 2005.

21. M. H. Frosz, P. Falk, and O. Bang, "The role of the second zero-dispersion wavelength in generation of supercontinua and bright-bright soliton-pairs across the zero-dispersion wavelength: erratum," Opt. Express 15(8), pp. 5262-5263, 2007.

22. M. H. Frosz, P. Falk, and O. Bang, "Supercontinuum generation in a photonic crystal fiber with two zerodispersion wavelengths tapered to normal dispersion at all wavelengths," Opt. Express 13(19), pp. 7535$7540,2005$.

23. G. Genty, M. Lehtonen, and H. Ludvigsen, "Effect of cross-phase modulation on supercontinuum generated in microstructured fibers with sub-30 fs pulses," Opt. Express 12(19), pp. 4614-4624, 2004.

24. A. Efimov, A. J. Taylor, F. G. Omenetto, A. V. Yulin, N. Y. Joly, F. Biancalana, D. V. Skryabin, J. C. Knight, and P. S. Russell, "Time-spectrally-resolved ultrafast nonlinear dynamics in small-core photonic crystal fibers: Experiment and modelling," Opt. Express 12(26), pp. 6498-6507, 2004. 
25. T. Schreiber, T. V. Andersen, D. Schimpf, J. Limpert, and A. Tünnermann, "Supercontinuum generation by femtosecond single and dual wavelength pumping in photonic crystal fibers with two zero dispersion wavelengths," Opt. Express 13(23), pp. 9556-9569, 2005.

26. J. M. Dudley, G. Genty, and S. Coen, "Supercontinuum generation in photonic crystal fiber," Rev. Mod. Phys. 78(4), pp. 1135-1176, 2006.

27. M. H. Frosz, O. Bang, and A. Bjarklev, "Soliton collision and Raman gain regimes in continuous-wave pumped supercontinuum generation," Opt. Express 14(20), pp. 9391-9407, 2006. 